\title{
On a strong coupling property of QCD
}

\author{
T. Grandou ${ }^{1, a}$ \\ ${ }^{1}$ Institut Non-Linéaire de Nice Sophia-Antipolis, UMR-CNRS 7335, 06560 Valbonne, France
}

\begin{abstract}
The fermionic Green's functions of QCD exhibit an unexpected property of effective locality, which appears to be exact, involving no approximation. In the limit of strong coupling, and at eikonal and quenching approximations (where this property was first discovered), effective locality implies a dependence of non-perturbative fermionic Green's functions on the full algebraic content of the rank 2-S $U_{c}(3)$ color algebra. At variance with Perturbation Theory and a variety of non-perturbative approaches also, $C_{3}$-dependences show up, where $C_{3}$ stands for the second, trilinear Casimir invariant of $S U_{c}(3)$. These dependences are sub-leading in magnitude and seem to comply with the maximally allowed departures from the pure $C_{2}$ behaviours advocated by lattice numerical estimates.
\end{abstract}

\section{Introduction}

A few years ago, a property bearing on the non-perturbative fermionic Green's functions of QCD has been discovered and dubbed effective locality [1]. Of course, Non-perturbativeness, being a negative notion, it admits a variety of quite different realizations. Here, it refers to the fact that the gluonic degrees of freedom, inherently perturbative, are fully integrated upon. In words, the property of effective locality can be phrased as follows.

For any Quark/Quark (or Anti-Quark) 2n-point Green's function and related amplitudes, the full gauge-fixed sum of cubic and quartic gluonic interactions, fermionic loops included, results in a local contact-type interaction. This local interaction is mediated by a tensorial field structure which is antisymmetric both in Lorentz and color indices. Moreover, the resulting sum is explicitly gaugefixing independent.

This is a non-expected result because, ordinarily, integrations of elementary degrees of freedom result in highly non-local structures. The 'effective locality' denomination, which sounds like an oxymoron, accounts for this rather unusual circumstance.

The previous definition of effective locality must be amended as follows.

1-The statement above holds true for the whole family of physical, ghost-free gauges, propagating the two degrees of freedom of the gluonic fields (planar, axial-planar, Fock-Schwinger gauges,..).

2- In a covariant quantization of QCD, effective locality can also be derived, in the same way as in the ghost free case, at least formally [2]. However, effective locality appears to be more tricky an

\footnotetext{
ae-mail: thierry.grandou@inln.cnrs.fr
} 
issue as soon as one sticks to the standard framework of BRST symmetry, whose definite perturbative character has been recognized [3]. Yet, a more involved treatment [2] allows one to recover the property of effective locality, by restoring the manifest gauge-invariance which is broken at the level of the Halpern representation (See Eq.(6) below).

Through this point, the very non-perturbative character of effective locality shows up in a certain manner. Contrarily to what could be proven in the pure YM case [4], effective locality doesn't stand for an expression of QCD that would be dual to the original one. Like the YM dual formulation of [4], though, effective locality seems to describe a genuine non-perturbative aspect of QCD. And this, not solely because, seen from Perturbation Theory, it would correspond to the sum of any possible non abelian interactions between scattering quarks, still an enumerable sum, but more specifically, because the resulting expression can in no way be reduced to any perturbative summation, even infinite. This cannot be illustrated in a more cogent way than by pointing out that in one case, scattering is mediated by non-local vectorial gluon exchanges, whereas in the other case, it is locally mediated by an involved tensorial structure, antisymmetric both in colour and Lorentz indices.

3-By gauge-fixing independence, the following is meant. As in Perturbation Theory, non-perturbative calculations are ordinarily performed by explicitly breaking gauge invariance. This is achieved by selecting a particular gauge, and if dully carried out, results shouldn't depend on that choice, so far as observables are concerned.

In the derivation of effective locality, the gauge-fixing procedure also appears appropriate in order to make mathematical sense of some intermediate steps to be taken. However, the crucial point is that, whatever a gauge-fixing being adopted, the functional expressions displaying the property of effective locality remain the same. At a formal level at least, this means that these expressions are gauge-fixing independent, and indeed, fully gauge-invariant by construction [2]. This exceptional situation appears to be deeply related to the fact that within the non-perturbative regime, interactions would no longer be mediated by the original gluonic fields, and correlatively, that gluonic degrees of freedom should no longer be taken as relevant, enumerable ones. A review article on the matter will be presented elsewhere [2]. Furthermore, as a decisive advantage, no systematic uncertainty related to Gribov's copies is met because, instead of functional integrations, the quantization procedure is achieved by means of functional differentiations in a fully equivalent way [2].

One may observe that truncated to the (euclidean) pure Yang Mills theory (YM), our nonperturbative QCD results can be compared to those obtained in [4], where a formulation dual to the original pure YM theory could be achieved at first non-trivial orders of an expansion in powers of $\hbar$. As well known, though, apart from a supersymmetric extension, effective locality cannot stand for a formulation that would be dual to the original full QCD theory. But as in the YM truncation, it remains that aspects of duality are somewhat displayed within the effective locality context.

Be it as it may, if effective locality is a sound property of non-perturbative QCD, then its consequences, even examined "at tree level" should exhibit admissible as well as new aspects of the strong coupling regime of QCD; and so far, it seems to be so [5]. In this communication we would like to report on a new aspect of the non-perturbative regime of QCD, such as revealed by the property of effective locality.

In [6], a general form of the non-perturbative QCD fermionic Green's functions is displayed as an $O_{N}(\mathbb{R})$-averaged finite sum of finite products of Meijer special functions (See Eq.(18)), in agreement with general expectations [7]. Remarkably also, within one and the same expression, these Green's functions are able to display an explicit link between a partonic content and a hadronic nonperturbative component in accord, this time, with the $A d S_{5} / Q C D$ light-cone approach of [8]. 
Based on [6], our results make use of the simplifying eikonal and quenching (i.e., the fermionic determinant is taken to 1) approximations. While the former has long been known to be most appropriate to the study of high energy scattering processes, the latter does not preserve unitarity. In Perturbation Theory, unitarity usually goes along with some compensation mechanisms. It could therefore happen that, if not washed out, our present results be somewhat modified by a restoration of unitarity, that is by relaxing the quenching approximation. First inspections, though, seem to maintain the essence of the quenched results to be exposed below [9].

\section{Effective Locality in short}

The current context is the one of Lagrangian Quantum Field Theory, and up to a few adaptations to be introduced shortly, the steps leading to the property of effective locality are those of standard functional methods.

\subsection{Elements of formalism}

As three basic elements, one has the gluon propagator,

$$
D_{F, \mu \nu}^{a b(\zeta)}(k)=\frac{i \delta^{a b}}{k^{2}+i \varepsilon}\left[g_{\mu \nu}-\zeta k_{\mu} k_{v} / k^{2}\right], \zeta=\lambda /(1-\lambda),
$$

corresponding to the covariant gauge-fixing lagrangian term of $\frac{\lambda}{2}\left(\partial \cdot A^{a}\right)^{2}$. However, for the reasons evoked in the previous section, and fully analyzed in [2], Fadeev-Popov ghost-fields will not be considered. The quark field propagator in an external gluon field $A_{\mu}^{a}$ is given by,

$$
G_{F}(x, y \mid A)=\left\langle x\left|\left[i \gamma^{\mu}\left(\partial_{\mu}-i g A_{\mu}^{a} \lambda_{a}\right)-m\right]^{-1}\right| y\right\rangle,
$$

with the Lie algebra generators, the $\lambda_{a}$, taken in the relevant representation of $S U_{c}(3)$. Eventually, the gauge-invariant closed fermion loop functional reads,

$$
L[A]=\operatorname{Tr} \ln \left[1-i g(\gamma \cdot A \cdot \lambda) S_{F}\right], \quad S_{F}=G_{F}[g A=0] .
$$

Not so standard is the following identity, in terms of functional differentiations,

$$
\mathcal{F}\left[\frac{1}{i} \frac{\delta}{\delta j}\right] e^{\frac{i}{2} \int j \cdot D_{F}^{(\zeta)} \cdot j}=e^{\frac{i}{2} \int j \cdot D_{F}^{(\zeta)} \cdot j} e^{\mathbf{D}_{A}^{(\zeta)}} \mathcal{F}[A]_{\left.\right|_{A=\int D_{F}^{(\zeta)} \cdot j}}
$$

with almost no constraints bearing on the very form of the functional $\mathcal{F}[10]$, and where $\mathbf{D}_{A}^{(\zeta)}$ stands for the so-called linkage operator,

$$
\mathbf{D}_{A}^{(\zeta)}=-\left.\frac{i}{2} \int \mathrm{d}^{4} x \int \mathrm{d}^{4} y \frac{\delta}{\delta A_{\mu}^{a}(x)} \mathbf{D}_{F}^{(\zeta)}\right|_{\mu \nu} ^{a b}(x-y) \frac{\delta}{\delta A_{v}^{b}(y)} .
$$

For this latter operator to be efficient, one needs to linearize the original quartic $A_{\mu}^{a}$-dependences of the QCD Lagrangian density. This can be achieved by using the Halpern trick,

$$
e^{-\frac{i}{4} \int \mathbf{F}^{2}}=\mathcal{N} \int \mathrm{d}[\chi] e^{\frac{i}{4} \int \Sigma\left(\chi_{\mu \nu}^{a}\right)^{2}+\frac{i}{2} \int \chi_{a}^{\mu \nu} \mathbf{F}_{\mu \nu}^{a}}
$$

In terms of these elementary definitions and using (4), the Green's function generating functional can be expressed as,

$$
3[j, \bar{\eta}, \eta]=\left.\mathcal{N}^{\frac{i}{2} \int j \cdot D_{F}^{(\zeta)} \cdot j} \int \mathrm{d}[\chi] e^{\frac{i}{4} \int \chi^{2}} e^{\mathfrak{D}_{A}^{(\zeta)}} \cdot e^{-\frac{i}{2} \int \chi \cdot \mathbf{F}+\frac{i}{2} \int A \cdot D_{F}^{(\zeta)-1} \cdot A} \cdot e^{i \int \bar{\eta} \cdot \mathbf{G}_{\mathrm{F}}[A] \cdot \eta+\mathbf{L}[A]}\right|_{A=\int \mathbf{D}_{\mathrm{F}}^{(\zeta)} \cdot j}
$$




\subsection{Fradkin's}

Again, for the linkage operator (5) to act in an efficient way, one needs convenient representations for the $A_{\mu}^{a}$-field functionals of (2) and (3). In the case of (2), one has for example the Fradkin's (mixed) representation,

$$
\begin{aligned}
\left\langle p\left|\mathbf{G}_{F}[A]\right| y\right\rangle= & e^{-i p \cdot y} i \int_{0}^{\infty} \mathrm{d} s e^{-i s m^{2}} e^{-\frac{1}{2} \operatorname{Tr} \ln (2 h)} \\
& \times \int \mathrm{d}[u]\{m-i \gamma \cdot[p-g A(y-u(s))]\} e^{\frac{i}{4} \int_{0}^{s} \mathrm{~d} s^{\prime}\left[u^{\prime}\left(s^{\prime}\right)\right]^{2}} e^{i p \cdot u(s)} \\
& \times\left(e^{g \int_{0}^{s} \mathrm{~d} s^{\prime} \sigma \cdot \mathbf{F}\left(y-u\left(s^{\prime}\right)\right)} e^{-i g \int_{0}^{s} \mathrm{~d} s^{\prime} u^{\prime}\left(s^{\prime}\right) \cdot \mathbf{A}\left(y-u\left(s^{\prime}\right)\right)}\right)_{+},
\end{aligned}
$$

with $h\left(s_{1}, s_{2}\right)=s_{1} \Theta\left(s_{2}-s_{1}\right)+s_{2} \Theta\left(s_{1}-s_{2}\right), \quad h^{-1}\left(s_{1}, s_{2}\right)=\frac{\partial}{\partial s_{1}} \frac{\partial}{\partial s_{2}} \delta\left(s_{1}-s_{2}\right)$, and where the boldface expressions stand for Lie-algebra valued $A$ and $F$ fields. The 4-vector $u(s)$ is the Fradkin variable. In the last line of (8), the +-subscript indicates an $s^{\prime}$-Schwinger-proper-time ordering of the expression between parenthesis. Fradkin's representations cannot be thought of as being of a very simple usage. But they are exact. Recently, they have even been used to re-derive some standard results of Quantum Mechanics in a certainly involved, still successful way [11].

- Plugged into (7), a representation like (8) produces a cumbersome structure of an exponential of an exponential. This is why it is necessary to bring (8) down by means of functional differentiations with respect to the Grassmannian sources $\bar{\eta}, \eta$, and deal with $2 n$-point fermionic Green's functions. Accordingly, the property of effective locality is readable on fermionic Green's functions rather than on their generating functional itself. Of course, there is no restriction in this because it is precisely out of the latter that the whole physical content of the theory is obtained.

- Yet, this is not enough because the linear and quadratic $A_{\mu}^{a}$-field dependences are comprised within a time-ordered exponential which prevents the linkage (5) to operate in a simple way.

- At the expense of introducing 2 extra field variables, it is possible to take the $A_{\mu}^{a}$-field variables out of the ordered exponential of (8). For example, this can be achieved by writing,

$$
\left(e^{i g p^{\mu} \int_{-\infty}^{+\infty} \mathrm{d} s A_{\mu}^{a}(y-s p) T^{a}}\right)_{+}=\mathcal{N} \int \mathrm{d}[\alpha] \int \mathrm{d}[\Omega] e^{-i \int_{-\infty}^{+\infty} \mathrm{d} s \Omega^{a}(s)\left[\alpha^{a}(s)-g p^{\mu} A_{\mu}^{a}(y-s p)\right]}\left(e^{i \int_{-\infty}^{+\infty} \mathrm{d} s \alpha^{a}(s) T^{a}}\right)_{+}
$$

in the case of an eikonal approximation of (8) which will be used from now on. Likewise, as advertised in the Introduction, the approximation of quenching is used also, and this is why the Fradkin's representation of the functional $L(A)$ of (7) can be ignored.

However, not until the full integrations on the 2 extra field variables $\alpha^{a}$ and $\Omega^{a}$ are brought to completion, can one be insured to deal with the proper Fradkin representation of $G_{F}[A]$. Fortunately enough, this is one of the most favourable circumstances encountered in these calculations: Integrating these extra dependences can be carried out in an exact way.

\subsection{Staring at effective locality at eikonal and quenching approximations}

It is in the framework of these 2 simplifications of QCD, eikonal and quenching, that effective locality was first observed [5], before being extended to the full non-approximated theory where it takes the form of a longer functional statement [1].

Illustrating things on the basis of a 4-point fermionic Green's function, two propagators $G_{F}\left(x_{1}, y_{1} \mid A\right)$ and $G_{F}\left(x_{2}, y_{2} \mid A\right)$ are to be represented with the help of (9). Omitting for short integrations on Schwinger proper times, Fradkin's variables and the 4 extra fields $\alpha_{i}^{a}$ and $\Omega_{i}^{a}, i=I$, II, one 
gets a result of

$$
\left.\prod_{i=I}^{I I} \int \mathrm{d} s_{i} \int \mathrm{d} u_{i}\left(s_{i}\right) \int \mathrm{d} \alpha_{i}\left(s_{i}\right) \int \mathrm{d} \Omega_{i}\left(s_{i}\right)(\ddots) \int \mathrm{d}[\chi] e^{\frac{i}{4} \int \chi^{2}} e^{\mathfrak{D}_{A}^{(\zeta)}} e^{+\frac{i}{2} \int A_{\mu}^{a} K_{a b}^{\mu v} A_{\nu}^{b}} e^{i \int Q_{\mu}^{a} A_{a}^{\mu}}\right|_{A \rightarrow 0}
$$

where,

$$
K_{a b}^{\mu v}=g f^{a b c} \chi_{c}^{\mu \nu}+g^{\mu v} \delta_{a b} D_{F}^{(\zeta)-1}, \quad Q_{\mu}^{a}=-\partial^{v} \chi_{\mu \nu}^{a}+g\left[R_{I \mu}^{a}+R_{I \mu \mu}^{a}\right], \quad f^{a b c} \chi_{\mu \nu}^{c} \equiv(f \cdot \chi)_{\mu \nu}^{a b} .
$$

Now, the action of the linkage operator on the last two terms of (10), followed by the prescription of cancelling the $A_{\mu}^{a}$ fields, produces a result,

$$
\mathcal{N} \int \mathrm{d}[\chi] e^{\frac{i}{4} \int \chi^{2}}[\operatorname{det}(g f \cdot \chi)]^{-\frac{1}{2}} e^{-\frac{i}{2 g} \int \mathrm{d}^{4} z Q(z) \cdot[(g f \cdot \chi)]^{-1}(z) \cdot Q(z)}
$$

where the locality of the effective interaction is manifest and mediated by the structure of $[g f \cdot \chi]^{-1}$. This offers a simple way to look at the effective locality phenomenon, whose detailed derivation relies in an essential way on the non-abelian character of the gauge group. It cannot show up in the QED abelian case, contrary to some long related expectations [12]. As mentioned in the Introduction, in the pure YM case, a form of effective locality was observed also, as a welcome property in an attempt to define a dual expression of the YM theory [4].

\section{Color algebraic structure of fermionic Green's functions}

In the framework of eikonal and quenching approximations at least, effective locality discloses an algebraic property of non-perturbative fermionic Green's function which is at variance with perturbative as well as a series of non-perturbative calculations [9]. In effect, while the latter display overall dependences of fermionic Green's functions on the first Casimir operator alone, $C_{2}=\sum_{a} T_{a}^{2}$, effective locality reveals an extra, sub-leading dependence on the full algebraic content of the rank-2 $S U_{c}$ (3)-Lie algebra, that is, an extra dependence on the tri-linear invariant $C_{3}=\sum d_{a b c} T^{a} T^{b} T^{c}$.

\subsection{QCD amplitudes by Random Matrix}

It is thanks to the property of effective locality that use can be made of the measure image theorem [13] in order to pass from an infinite dimensional functional integration,

$$
\int \mathrm{d}[\chi] \equiv \prod_{x \in \mathcal{M}} \prod_{a=1}^{N^{2}-1} \prod_{0=\mu<\nu}^{3} \int \mathrm{d}\left[\chi_{\mu \nu}^{a}\right](x)
$$

to ordinary Lebesgue integrations over finite-dimensional linear spaces. Constructing $M$ the matrix,

$$
\sum_{a=1}^{N_{c}^{2}-1} \chi_{\mu \nu}^{a} \otimes T_{a d j}^{a}=i M, \quad M_{i j}=M_{j i} \in \mathbb{R}, \quad 1 \leq i, j \leq N, \quad \operatorname{Tr} M=0, \quad N=D \times\left(N_{c}^{2}-1\right),
$$


effective locality enables the passage to (a standard analytic continuation of) Random Matrix integration with measure [14],

$$
\begin{aligned}
& \mathrm{d}\left(\sum_{a=1}^{N_{c}^{2}-1} \chi_{\mu \nu}^{a} \otimes T^{a}\right) \\
= & i \mathrm{~d} M=i \mathrm{~d} M_{11} \mathrm{~d} M_{12} \cdots \mathrm{d} M_{N N} \\
= & i\left|\frac{\partial\left(M_{11}, \cdots, M_{N N}\right)}{\partial\left(\xi_{1}, \cdots, \xi_{N} ; p_{1}, \cdots, p_{N(N-1) / 2}\right)}\right| \mathrm{d} \xi_{1} \cdots \mathrm{d} \xi_{N} \mathrm{~d} p_{1} \cdots \mathrm{d} p_{N(N-1) / 2} \\
= & i \prod_{i=1}^{N} \mathrm{~d} \xi_{i} \prod_{i<j}\left|\xi_{i}-\xi_{j}\right|^{k=1} \mathrm{~d} p_{1} . . \mathrm{d} p_{N(N-1) / 2} F(p) .
\end{aligned}
$$

Measure (15) factorizes into a measure over a spectrum of pairwise opposite real eigenvalues,

$$
\operatorname{Sp} M=\left\{\left(\xi_{i}, \xi_{N-i+1}=-\xi_{i}\right)\right\}, \quad-\infty \leq \xi_{i} \leq+\infty, \quad \forall i=1,2, \ldots, N / 2,
$$

and an integration over the orthogonal group $O_{N}(\mathbb{R})$, endowed with some Haar measure $\mathrm{d} p_{1} . . \mathrm{d} p_{N(N-1) / 2} F(p)$.

Then, the strong coupling limit of $g>>1$ is introduced; not because non-perturbativeness would be intrinsically related to it [15], but because it allows one to drop the $\partial^{v} \chi_{\mu \nu}^{a}$-term of (11) that so far we have been unable to treat on the same footing as the other terms.

Within this framework, one can calculate the non-perturbative $2 n$-point fermionic Green's functions in a generic closed form, a result which complies with a general conjecture stating that all Quantum Field Theory Green's functions are expandable in terms of $G_{p q}^{m n}$-Meijer's special functions, [7]. Integrating over the eigenvalue spectrum (16) produces the expected Meijer special functions,

$$
\int_{0}^{\infty} \mathrm{d} \xi_{i} \xi_{i}^{p} e^{-\xi_{i}^{2}-\frac{b_{i}}{\xi_{i}}}=\frac{1}{2 \sqrt{\pi}} G_{03}^{30}\left(\frac{b_{i}^{2}}{4} \mid \frac{p+1}{2}, \frac{1}{2}, 0\right)
$$

and while the left hand side of (17) is only defined at $p>0$ and $b_{i}>0$, another most favourable circumstance of these calculations is that the right hand side turns out to be analytic both in the argument and parameters, $b_{i}$ and $p$.

\subsection{Colour algebraic dependences at strong coupling}

Eventually, it is by integrating over the orthogonal group $O_{N}(\mathbb{R})$ that the full content of the $S U_{c}(3)$ colour algebra is revealed. In effect, exact integrations over Fradkin's variables, the spectrum (16) and the auxiliary fields $\alpha_{i}^{a}$ and $\Omega_{i}^{a}$ in (9) can be carried out with a result of [9],

$$
\begin{aligned}
& \sum_{\text {monomials }}\left\langle\prod_{1 \leq i \leq N}^{\sum q_{i}=N(N-1) / 2}\left[1-i(-1)^{q_{i}}\right]\right. \\
\times & {\left[\frac{\sqrt{2 i N_{c}} \sqrt{\widehat{s}\left(\widehat{s}-4 m^{2}\right)}}{m^{2}}\right] \frac{\left[(O \mathcal{T})_{i}\right]^{-2}}{g \varphi(b)} } \\
\times & \left.G_{03}^{30}\left(\left[\frac{g \varphi(b)}{\sqrt{32 i N_{c}}} \frac{m^{2}}{\sqrt{\widehat{s}\left(\widehat{s}-4 m^{2}\right)}}\right]^{2}\left[(O \mathcal{T})_{i}\right]^{4} \mid \frac{1}{2}, \frac{3+2 q_{i}}{4}, 1\right)\right\rangle_{O_{N}(\mathbb{R})},
\end{aligned}
$$


where the overall big brackets are here to remind that an $O_{N}(\mathbb{R})$ average is in order. At this level of approximations at least, the structure of the result is that of an $O_{N}(\mathbb{R})$-averaged finite sum of finite products of Meijer functions. This result is non-trivial. It is due to properties of the $O_{N}(\mathbb{R})$ average which allows one to recover, in the non-perturbative situation, a structure which is that of the Wick theorem for $T$-products of field operators [9]. In (18) one has the scattering process sub-energy $\widehat{s}=$ $\left(p_{1}+p_{2}\right)^{2}$, where $p_{1}$ and $p_{2}$ are the 2 scattering quarks 4-momenta, and $m$ their mass (one quark flavour only is considered). The function $\varphi(b)=(\mu / \sqrt{\hat{s}}) e^{-(\mu b)^{2-\xi}}$ is non-perturbative, depending on $b$ the impact parameter of the 2 scattering quarks in their $\mathrm{cms}$ frame of reference, whereas $\mu$ is that mass scale necessarily introduced by the property of effective locality [6]. Small and positive, $\varepsilon$ is a deformation parameter essential to a description of quark confinement in terms of an almost linear potential, while it seems connected also to other possible aspects of the QCD non-perturbative regime, such as lowest lying Landau levels, Levy flights mode of quark propagation, and a non-commutative geometry of the transverse scattering planes, i.e., de Moyal planes. There is some flexibility in the definition of $\varphi(b)$ with no incidence at all on the colour algebraic dependences of the results. Average is taken with respect to $O \in O_{N}(\mathbb{R})$, acting upon an $N$-vector $\mathcal{T}$ made out of $D=4$ copies of generators of the $S U_{c}(3)$ algebra taken in the fundamental representation (i.e., 4 copies of the Gell-Mann set of matrices $\left.\left\{\lambda_{1} / 2, \lambda_{2} / 2, \ldots, \lambda_{8} / 2\right\}\right)$. Some flexibility exists also in the choice of the Haar measure $F(p)$ on $O_{N}(\mathbb{R})$ with, again, no incidence at all on the results which only depend on two basic properties of these measures [9].

Then, defining,

$$
z_{i} \equiv \lambda\left[(\mathcal{O T})_{i}\right]^{4}, \quad \lambda \equiv\left(\frac{g \varphi(b)}{\sqrt{32 i N_{c}}} \frac{m^{2}}{\sqrt{\widehat{s}\left(\widehat{s}-4 m^{2}\right)}}\right)^{2},
$$

the matrix-valued argument of the $G_{03}^{30}$ - Meijer function, one has $\left|z_{i}\right|<<1$ even at strong enough coupling $(g=15-20)$, a moderate scattering sub-energy of $\sqrt{\widehat{s}}=100 \mathrm{MeV}$ and a current light quark mass parameter of $m=5 \mathrm{MeV}$, at a non-perturbative input of $\varphi(b) \simeq 1$. Expanding $G_{03}^{30}$ in powers of $z_{i}$, one finds a leading order algebraic dependence of,

$$
\forall i, 1 \leq i \leq N, \quad<\sqrt{z_{i}}>_{O_{N}(\mathbb{R})}=\frac{\sqrt{\lambda}}{N} D C_{2 f} \mathbf{1}_{3 \times 3},
$$

where $C_{2 f}$ is the quadratic Casimir operator eigenvalue on the fundamental representation, $C_{2 f}=$ $\sum_{a=1}^{8}\left(\lambda_{a} / 2\right)^{2} \equiv C_{F}=4 / 3$. It is at next to leading order that the full algebraic content of the rank 2 $S U_{c}(3)$-Lie algebra shows up, with,

$$
\forall i, 1 \leq i \leq N, \quad<z_{i}>_{O_{N}(\mathbb{R})}=\left(\frac{\sqrt{\lambda}}{N}\right)^{2}\left(\left(D C_{2 f}\right)^{2}+\left(D C_{3 f}\right)\right) \mathbf{1}_{3 \times 3},
$$

where, at $N_{c}=3$ and $t_{a} \equiv \lambda_{a} / 2$,

$$
\left\{t_{a}, t_{b}\right\}=d_{a b c} t_{c}+\frac{1}{3} \delta_{a b}, \quad \sum_{a, b, c=1}^{N_{c}^{2}-1} d_{a b c} t^{a} t^{b} t^{c} \equiv C_{3 f} \mathbf{1}_{3 \times 3}, \quad C_{3 f}=10 / 9 .
$$

And likewise, at sub-sub-leading order, a much longer calculation yields, for all $i, 1 \leq i \leq N$,

$$
\begin{aligned}
& <z_{i} \sqrt{z_{i}}>_{O_{N}(\mathbb{R})}= \\
& \left(\frac{\sqrt{\lambda}}{N}\right)^{3}\left(\left[2+\left(\frac{5}{6}\right)^{2}\right]\left(D C_{2 f}\right)^{2}+\left(D C_{2 f}\right)\left(D C_{3 f}\right)+3\left(D C_{3 f}\right)\right) \mathbf{1}_{3 \times 3} .
\end{aligned}
$$


At sub-leading order (21) displays, due to $C_{3 f}$, an enhancement of the sole $C_{2 f}$ contribution an amount of $15.6 \%$ that can be compared to the $15 \%$ at most, advocated in [16], whereas at sub-sub-leading order (23), $C_{2 f}$ and $C_{3 f}$ contributions are identical to within $0.2 \%$. That is, so long as sub-leading orders are concerned, elementary $C_{3 f}$ contributions cannot be taken as negligible ones.

Now the question is indeed to know how much is (21) reflected at the whole level of (18). This is a long numerical affair about which two insights may be proposed. Selecting out of (18) the monomial with a $q_{i}$-power distribution such as $(N-1, N-2, \ldots, 1,0)$, one can calculate the relative deviation to a pure $C_{2 f}$ behaviour (20),

$$
\delta_{C}=\frac{\sqrt{\lambda}}{N} \frac{D C_{3 f}+\left(D C_{2 f}\right)^{2}}{D C_{2 f}} \frac{\sum_{1}^{N} b_{i}+\sum_{2}^{N} a_{i} \sum_{1}^{i-1} a_{j}}{\sum_{1}^{N} a_{i}}+O\left(\left(\frac{\sqrt{\lambda}}{N}\right)^{2}\right),
$$

where one has [9],

$$
a_{i} \equiv-2 \Gamma\left(\frac{2 q_{i}-1}{4}\right) / \Gamma\left(\frac{2 q_{i}+1}{4}\right), \quad b_{i} \equiv 8 /\left(2 q_{i}-3\right) .
$$

One finds, $\delta_{C}(N-1, N-2, \cdots, 1,0) \simeq-1.0 \%$, a result obviously in line with the $5 \%$ of deviation allowed by lattice simulations. There are of course a very large number of similar contributions. Another indication may be provided by the much simpler case of $N=4$. Then the sum of all monomials yields a departure which, even at a relatively large estimate of the expansion parameter $\lambda,(19)$, reads as,

$$
-\frac{1798,211444}{147,543465} \frac{\sqrt{\lambda}}{4} \frac{D C_{3 f}+\left(D C_{2 f}\right)^{2}}{D C_{2 f}}=-7.5 \% \text {. }
$$

This result is acceptable also, keeping in mind the approximated context of our calculations, on the one hand, and in the other hand, the fact that dynamical quarks are here dealt with, instead of the somewhat 'academic' static quarks of lattice simulations.

\section{Conclusion}

Even though approximated, that is obtained at strong coupling, eikonal and quenching approximations, these estimates disclose an overlooked dependence of non-perturbative fermionic Green's functions on the second Casimir operator of the $S U_{c}(3)$-Lie algebra, which is effectively rank 2 an algebra. In the absence of any super-selection rule that would keep $C_{3 f}$ to appear, one may think that, in a non-perturbative context, such a full dependence on the $S U_{c}(3)$-Lie algebra content had to come about, soon or later. These dependences occur in a quite sub-leading way in agreement with boundaries obtained through lattice simulations, and do not seem to be compromised by a relaxation of the quenching approximation. Also, they are obtained for dynamical rather than static quark fields.

These results, are non-perturbative in several respects, practical $(g>>1)$ and theoretical (gluons integrated out), and a consequence of effective locality which is itself a non-perturbative property of the fermionic Green's functions of QCD. Of course, it would be worth improving on them, and the more so as, if lengthy, this is doable.

Now, at a more fundamental level, it matters also to make sense of this intriguing and unexpected formal property of effective locality, such as displayed by the non-perturbative fermionic field functions of QCD [2]. In particular the manner in which non abelian gauge invariance gets realized in the non-perturbative regime, and the meaning of the mass scale effective locality necessarily requires in order to avoid triviality. 


\section{References}

[1] H.M., Fried, T. Grandou, and Y.-M., Sheu Ann. Phys. 327, 2666-2690 (2012).

[2] H.M. Fried, T. Grandou and R. Hofmann, work in progress.

[3] C. Becchi, in http://www.scholarpedia.org/article/Becchi-Rouet-Stora-Tyutin-symmetry.

[4] H. Reinhardt, K. Langfeld and L. v. Smekal, Phys. Lett. B, 300, 11 (1993); H. Reinhardt, "Dual description of QCD", arXiv:hep-th/9608191v1 (1996).

[5] H.M. Fried, Y. Gabellini, T. Grandou and Y.-M. Sheu, Eur. Phys. J. C65, 395 (2010). H.M. Fried, Y. Gabellini, T. Grandou and Y.M. Sheu, Ann. Phys. 338, 107-122 ( 2013). H.M. Fried, P.H.Tsang, Y. Gabellini, T. Grandou and Y.-M. Sheu, Ann. Phys. 359, 1-19 (2015).

[6] H.M. Fried, T. Grandou and Y.-M. Sheu, Ann. Phys. 344C,78-96 (2014).

[7] D.D. Ferrante, G.S. Guralnik, Z. Guralnik and C. Pehlevan, BROWN-HET-1611 (2011); see also S. Gukov and E. Witten (2008).

[8] G.F. de Téramond and S.J. Brodsky, arXiv:1203.4025 [hep-ph] (2012) ; G.F. de Teramond, S.J. Brodsky and H.G. Dosch(2014).

[9] T. Grandou, EPL 107, 11001 (2014). H.M. Fried, T. Grandou and R. Hofmann, Int. J. of Mod. Phys. A, 31, Nos. $20 \& 21,1650120$ (25 pages) (2016).

[10] H.M. Fried, Functional Methods and Models (the MIT Press, Boston, 1971) p.210.

[11] K.V. Shajesh and K.A. Milton, arXiv:hep-th/0510103v1.

[12] A. Zee, Quantum Field Theory in a Nutshell, 2nd Edition, (Princeton University Press, 2010) p.189.

[13] G. W. Johnson and M.L. Lapidus, The Feynman Integral and Feynman's Operational Calculus, (Oxford University Press, Oxford 2000).

[14] M. L. Mehta, Random Matrices (Academic Press, 1967, New York and London) Theorem 3.2, p.38.

[15] G. Van Baalen, D. Kreimer, D. Uminsky and K. Yeats, Ann. Phys. 325 (2010) 300.

[16] F. Cooper, J.F. Dawson and B. Mihaila, Phys. Rev. D 78, 117901 (2008). 